\title{
Epilepsy patients treated with vigabatrin exhibit reduced ocular blood flow
}

\author{
S L Hosking, E J Roff Hilton, S J Embleton, A K Gupta
}

Br J Ophthalmol 2003;87:96-100

\begin{abstract}
Background/aim: Reduced cerebral blood flow and decreased glucose metabolism have been identified in epilepsy patients receiving vigabatrin. It is likely that such a change may extend to the eye and may be linked to previously reported irreversible visual field defects. The aim of this study was to determine whether patients who have undergone anti-epileptic drug (AED) therapy with vigabatrin have altered ocular haemodynamics.

Methods: The study cohort comprised 11 normal subjects (mean age 42.6 (SD 12.7) years and 17 epilepsy patients, of which 10 were either currently or previously treated with vigabatrin (38.6 (1 1.7) years) and seven were treated with AEDs excluding vigabatrin (46.0 (9.8) years). The three groups were matched at baseline for pulse rate, diastolic and systolic blood pressure, and intraocular pressure (IOP). At a single visit, the ocular blood flow analyser (OBFA; Paradigm Medical Instruments Inc, UT, USA) was used to measure pulsatile ocular blood flow (POBF) and pulse amplitude (PA) in each eye of all subjects. One way ANCOVA (with age as a covariate) was used to identify differences in POBF and PA between the groups. For the vigabatrin group only, Pearson's product moment correlation coefficient was used to explore potential interactions between ocular blood flow parameters and cumulative vigabatrin dose, duration, and maximum dose. Results: Both the vigabatrin treated epilepsy group and conventionally treated epilepsy group exhibited significantly reduced POBF $(p=<0.001, p=0.040)$ and $P A(p=<0.001, p=0.005)$ compared to normal subjects. Patients treated with vigabatrin exhibited a further reduction in POBF $(p=0.046)$ and PA $(p=0.034)$ compared to conventionally treated epilepsy patients. No significant correlations were found between drug dosage and POBF and PA for the vigabatrin treated epilepsy group.

Conclusions: A significant reduction in POBF and PA is apparent in epilepsy patients treated with AEDs when compared to normal subjects. A further reduction in POBF and PA is apparent between vigabatrin treated and conventionally treated patients. The reduction in ocular perfusion, which is more pronounced in patients previously treated with vigabatrin, may have implications in the impairment of visual function associated with the drug.
\end{abstract}

See end of article for authors' affiliations

Correspondence to: Sarah L Hosking, PhD, Neurosciences Research Institute, School of Life and Health Sciences, Aston University, Aston Triangle, Birmingham B4 7ET, UK; s.l.hosking@aston.ac.uk

Accepted for publication 22 July 2002

$\mathrm{T}$ he effect of anti-epileptic drug (AED) therapy on cerebral blood flow has long been considered and poses an important question as variations in blood flow and glucose metabolism in the brain may have subsequent effects on neuronal functioning and cognitive performance. To this end, several AEDs have been investigated, including carbamazepine, phenytoin, phenobarbital, sodium valproate, and vigabatrin, all of which have been shown to result in reduced cerebral metabolic rate for glucose and/or decreased blood flow. ${ }^{1-3}$

Visual disturbances in epilepsy are not uncommon. ${ }^{4}$ In particular, diplopia, blurred vision, and colour perception defects are associated with AED therapy. ${ }^{5}$ AEDs known to affect vision directly include carbamazipine, phenytoin, and vigabatrin. More specifically, disturbances in colour vision have been reported in patients undergoing treatment with carbamazepine and phenytoin. ${ }^{67}$ Visual deficits common to vigabatrin are more severe, occurring in up to one third of patients receiving the drug and include concentric peripheral visual field loss, colour vision disturbances, and reductions in contrast sensitivity and visual acuity..$^{713}$ Electrophysiological studies have also identified defects in electroretinal function resulting from vigabatrin ${ }^{9}$; ; these have been identified in both the outer and inner retina at the level of the Müller cell. ${ }^{15}$

Vigabatrin ( $\gamma$-vinyl GABA) is a novel drug that is used primarily in the management of partial seizures and infantile spasms. The drug works by increasing brain levels of the inhibitory neurotransmitter $\gamma$-aminobutyric acid (GABA) through acting as an irreversible inhibitor of GABAtransaminase. In a recent study, Spanaki et al identified decreased cerebral blood flow and reduced glucose metabolism as a result of vigabatrin therapy and reported a significant inverse correlation between cerebral spinal fluid (CSF) GABA levels and cerebral glucose metabolism. GABA has numerous roles in the brain, one of which includes vascular regulation. Specific GABA receptor sites have been demonstrated, both in vivo and in vitro, as being functionally viable in eliciting a vasodilatory response in the presence of GABA and muscimol, a GABA agonist. ${ }^{16}{ }^{17}$ In contrast, Kelly and McCulloch ${ }^{18}$ have shown, using a rat model, that the systemic administration of GABAergic agonists results in a reduction in cerebral glucose metabolism that is accompanied by a decrease in cerebral blood flow. It therefore appears that there are two potentially opposing GABAergic mechanisms influencing cerebral blood flow and metabolism; the cumulative product of which is reduced blood flow and depressed metabolism.

There are numerous cell types under GABAergic transmission including subpopulations of amacrine and horizontal cells, and retinal ganglion cells. ${ }^{19-21}$ There is evidence that vigabatrin causes a greater inactivation of GABA transaminase in the retina than in the brain..$^{22}$ In a rat model, both the spinal cord and brain show greater tolerance to the toxic action of vigabatrin compared to the retina. ${ }^{23}$ Thus, the effect of vigabatrin on cerebral blood flow may be reflected in ocular perfusion and further enhanced as a result of raised GABA levels. A reduction in retinal and choroidal blood flow will have a direct effect on the health and functioning of various GABAergic retinal neurons and related cell groups resulting in reduced retinal sensitivity. 
Table 1 Subject sample characteristics showing age, sex, and refractive error distributions for the normal subjects and vigabatrin treated (VGB) and conventionally treated (conv) epilepsy groups

\begin{tabular}{lllll}
\hline & & & & \\
& Age, mean (SD) & $\begin{array}{l}\text { Refraction (mean } \\
\text { sphere) }\end{array}$ & & Sex $(\mathrm{n})$ \\
\cline { 5 - 5 } & & & Male & Female \\
\hline Normal $(n=11)$ & $42.6(12.7)$ & $-0.36(2.0)$ & 5 & 6 \\
VGB $(n=10)$ & $38.6(11.7)$ & $0.29(0.6)$ & 5 & 5 \\
Conv $(n=7)$ & $46.0(9.8)$ & $0.31(1.0)$ & 3 & 4 \\
\hline
\end{tabular}

The purpose of this investigation was to determine whether epilepsy patients who have undergone AED therapy with vigabatrin have altered ocular haemodynamics compared to those who have never received the drug and to normal healthy volunteers.

\section{METHODS \\ Subjects}

The study cohort comprised three subject samples: 11 normal control subjects, 10 epilepsy patients either currently or previously treated with vigabatrin, and seven epilepsy patients treated with conventional AEDs excluding vigabatrin. Table 1 outlines the subject sample characteristics.

Epilepsy patients were recruited from epilepsy clinics of Birmingham University Queen Elizabeth Psychiatric Hospital, and Birmingham and City Hospital NHS Trust, Birmingham, UK. Patients were excluded if they had a history of ischaemic heart disease, hypertension, hypotension, diabetes mellitus, or multiple sclerosis. Each patient underwent a full ophthalmic examination and was excluded if they had glaucoma, intraocular pressure (IOP) above $22 \mathrm{~mm} \mathrm{Hg}$ (Goldmann applanation tonometry), significant cataract, or any other sight threatening disease.

Of the epilepsy patients, two patients had complex partial seizures alone, three had simple and complex partial seizures, five had simple and complex partial seizures which secondarily generalised, six had complex partial seizures with secondary generalisation, and one had simple partial seizures which secondarily generalised (ILAE classification ${ }^{24}$ ). Of the epilepsy patients who had previously, or were currently, receiving vigabatrin, the maximum dose received ranged from $500 \mathrm{mg} /$ day to $3500 \mathrm{mg} /$ day. The average drug duration was 7 (SD 3.6) years and the total cumulative dose ranged from $1277 \mathrm{~g}$ to $16425 \mathrm{~g}$ (mean 6046 (4753) g). None of the patients who had received vigabatrin were on monotherapy; concomitant AEDs included carbamazepine, sodium valproate, lamotrigine, keppra, and clobazam. The conventionally treated epilepsy patients also received a range of AEDs including carbamazepine, sodium valproate, lamotrigine, keppra, clobazam, phenytoin, phenobarbital, and gabapentin.

Normal control subjects had no history of ocular or neurological disease or surgery. Intraocular pressures were less than $22 \mathrm{~mm} \mathrm{Hg}$ and visual fields were normal (Humphrey field analyser 24-2 full threshold procedure). Normal subjects were required to have best corrected visual acuities of $6 / 9$ or better in both eyes. Each normal subject underwent a comprehensive ophthalmic examination to rule out abnormalities of the optic nerve head, macular region, anterior segment, and posterior pole. Both the normal and epilepsy groups were required to have astigmatism less than 2 dioptres cylinder and refractive error of less than 6 dioptres in the test eye.

The three subject samples were matched at baseline for systemic variables (pulse rate, diastolic, and systolic blood pressure) and for IOP. Ethics committee approval that conformed to the tenets of the Declaration of Helsinki was obtained for all experimental procedures, and approved by the institutional review board. Written informed consent was acquired from each participant before study enrolment.

\section{Experimental procedure}

Ocular blood flow measurements were carried out at approximately the same time of day for each subject to minimise possible diurnal fluctuations in blood flow and IOP. ${ }^{25}$ On the night before and the morning of the test, subjects were requested to follow a strict diet that avoided caffeine containing products, alcohol, and nicotine (in any form). Subjects were also requested to abstain from strenuous exercise and to relax for 15 minutes before blood flow measurements commenced. Systemic systolic and diastolic blood pressure was assessed using a sphygmomanometer. The ocular blood flow analyser (OBFA; Paradigm Medical Instruments Inc, UT, USA) was used to measure the pulsatile component of ocular blood flow $(\mathrm{POBF})$, pulse amplitude (PA), pulse rate (PR), and IOP in each eye of each subject.

\section{Ocular blood flow analyser}

The principles of the OBFA (formerly the ocular blood flow tonograph) have been described in detail elsewhere. ${ }^{26-28}$ Briefly, the OBF analyser measures the pulsatile component of ocular blood flow. Each contraction of the heart forces a bolus of blood into the eye via the ophthalmic artery which rapidly perfuses the retinal and ciliary vascular network. Blood flow to the eye comprises two components: a steady continuous component and a pulsatile component that varies according to the arterial pulse. As the pulsatile component varies, rhythmic fluctuations in ocular volume and IOP occur resulting in the measured PA. Using a mathematical model of the relation between ocular pressure and volume, the OBFA calculates ocular volume at each instant in time and uses a volume/time plot to derive POBF. ${ }^{26} \mathrm{POBF}$ is reported to represent $75 \%-85 \%$ of total ocular blood flow and is primarily constituted by the choroidal circulation with some influence from the retinal vasculature. ${ }^{29}{ }^{30}$ Previous studies have demonstrated good reliability of POBF measurements using the OBFA. ${ }^{31} 32$

\section{Statistical analysis}

One way analysis of covariance (ANCOVA) was used to determine whether there was a significant difference in the measured ocular and systemic variables between the three subject groups. In each case group was used as the between factor, age as the covariate, and the measured parameter as the continuous variable. Fisher's PLSD post hoc analysis was used to identify which groups differed from each other. A significant difference was said to have occurred if $\mathrm{p}<0.05$.

Pearson's product moment correlation coefficient $(r)$ was used to explore possible correlations between the ocular blood flow variables (POBF and PA) and drug duration, maximum vigabatrin dose, and cumulative vigabatrin dose.

\section{RESULTS}

ANOVA revealed a significant difference between the three groups for age and posthoc analysis revealed that both the vigabatrin treated and normal group were significantly 
Table 2 Mean values (SD) for each measured systemic and ocular parameter for the normal, vigabatrin treated (VGB), and conventionally treated (conv) epilepsy groups

\begin{tabular}{lllllll}
\hline & IOP $(\mathrm{mm} \mathrm{Hg})$ & PA $(\mathrm{mm} \mathrm{Hg})$ & POBF $(\mu \mathrm{l} / \mathrm{s})$ & PR $(/ \mathrm{minute})$ & Syst BP $(\mathrm{mm} \mathrm{Hg})$ & Diast BP $(\mathrm{mm} \mathrm{Hg})$ \\
\hline Normal & $12.4(4.3)$ & $4.13(0.82)$ & $19.66(4.84)$ & $64.23(6.92)$ & $119.2(13.6)$ & $76.9(7.3)$ \\
VGB & $13.3(3.0)$ & $2.62(0.70)$ & $13.89(3.9)$ & $61.30(7.55)$ & $114.2(14.2)$ & $75.0(12.5)$ \\
Conv & $14.3(2.0)$ & $3.32(1.04)$ & $16.77(3.30)$ & $65.00(10.30)$ & $109.5(12.2)$ & $66.8(10.89)$
\end{tabular}

Table 3 ANCOVA results for each measured systemic and ocular parameter comparing the normal, vigabatrin treated (VGB), and conventionally treated (conv) epilepsy groups

\begin{tabular}{|c|c|c|c|c|c|c|}
\hline & IOP (mm Hg) & $\mathrm{PA}(\mathrm{mm} \mathrm{Hg})$ & POBF $(\mu \mathrm{l} / \mathrm{s})$ & PR (/minute) & Syst BP $(\mathrm{mm} \mathrm{Hg})$ & Diast BP $(\mathrm{mm} \mathrm{Hg})$ \\
\hline Norm-VGB & 0.853 & $<0.001$ & $<0.001$ & 0.105 & 0.385 & 0.511 \\
\hline Norm-conv & 0.319 & 0.005 & 0.040 & 0.931 & 0.111 & 0.061 \\
\hline VGB-conv & 0.414 & 0.034 & 0.046 & 0.174 & 0.157 & 0.084 \\
\hline
\end{tabular}

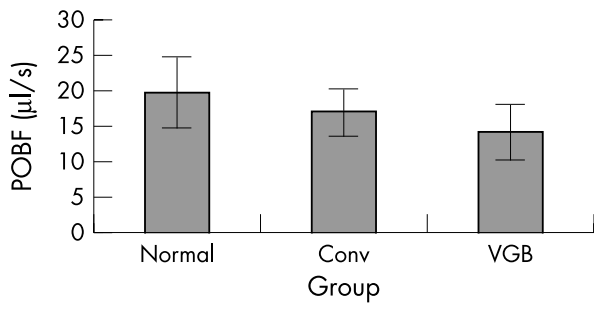

Figure 1 Mean values for POBF (pulsatile ocular blood flow) (SE) measured for the normal subject group, epilepsy group receiving conventional therapy (conv), and epilepsy group receiving vigabatrin (VGB).

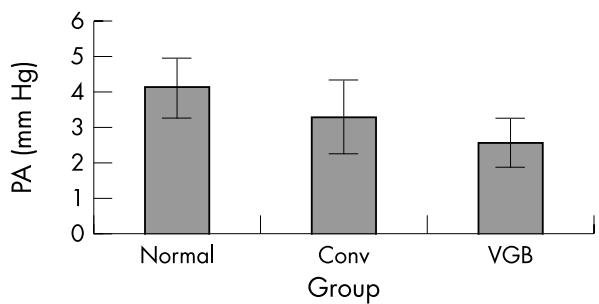

Figure 2 Mean values for PA (pulse amplitude) (SE) measured for the normal subject group, epilepsy group receiving conventional therapy (conv), and epilepsy group receiving vigabatrin (VGB).

younger than the conventionally treated epilepsy group $(\mathrm{p}=0.034$ and $\mathrm{p}=0.042$ respectively). As a result, age was incorporated as a covariate into the subsequent ANCOVA models, thus accounting for any potential age effect.

Table 2 gives the mean values for the ocular and systemic parameters. ANCOVA analysis revealed no significant difference between the three groups for blood pressure, pulse rate, or IOP $(\mathrm{p}<0.05)$. Table 3 gives the $\mathrm{p}$ values resulting from the ANCOVA analysis. POBF and PA were significantly lower for the vigabatrin treated epilepsy group compared to both the conventionally treated epilepsy group and the normal subject group (Figs 1 and 2).

No significant correlation was apparent between the ocular blood flow variables (POBF and PA) and vigabatrin maximum dose, cumulative dose, or duration of treatment $(p>0.05)$.

\section{DISCUSSION}

Overall, patients diagnosed with epilepsy exhibit a reduction in the pulsatile component of ocular blood flow and ocular pulse amplitude when compared to normal subjects; this finding may be a characteristic of epilepsy or AEDs not previously identified. A further reduction in POBF and PA was apparent in the vigabatrin treated epilepsy group when compared to normal control subjects and epilepsy patients previously treated with conventional drugs.

There are a number of possibilities that may explain the reduction in blood flow observed in the vigabatrin treated group. Firstly, vigabatrin may exert a direct effect on ocular perfusion. Decreased cerebral metabolic rate for glucose and blood flow has been previously reported in patients receiving vigabatrin and other AEDs. ${ }^{33}$ It is possible that the effect of AEDs on cerebral blood flow may be mediated via increased CSF GABA levels which are known to cause decreased metabolic rate for glucose and blood flow. ${ }^{18}$ The reason why a greater reduction in ocular blood flow was observed in vigabatrin treated patients when compared to the conventionally treated patients may be related to GABA levels. Vigabatrin causes a greater inactivation of GABA transaminase in the retina compared to the brain, ${ }^{22}$ the outcome of which is high localised GABA levels. The retina contains a number of GABAergic cell types that are likely to be affected by the raised GABA levels including subpopulations of amacrine and horizontal cells, interplexiform cells, Müller cells, and retinal ganglion cells. ${ }^{19-21}$ Hence, the raised GABA levels in the retina may result in a vascular response similar to the brain. POBF is a global measure of ocular blood flow with influences from both the choroidal and retinal circulations. While circulatory disturbances may have occurred in the retina, the choroid, which is also richly innervated, may also have been affected.

Another possibility that may explain the observed result is pre-existing ischaemia which may be attendant in a proportion of patients previously treated with vigabatrin. Our rationale for this is twofold: (1) a proportion of the general population present with ischaemic tendencies including vasospastic disorders such as cold extremities, peripheral vasospasm, migraine, and Reynaud's phenomenon. This is particularly relevant in low tension glaucoma where ischaemic tendencies are more prevalent. ${ }^{34}$ The proportion of patients presenting with vigabatrin associated visual field defects is not dissimilar to the proportion of glaucoma patients presenting with the low tension, vascular type; (2) optic nerve pallor, an ophthalmic sign known to be associated with ischaemia, has been identified in epilepsy patients treated with vigabatrin. ${ }^{86}{ }^{37}$ If a proportion of the epilepsy patients treated with vigabatrin were self selected for ischaemia then this would undoubtedly exacerbate the neurotoxic effects of vigabatrin in the retina. Increased neurotransmitter levels in the CNS have been identified in focal ischaemia ${ }^{38}$ and raised glutamate levels have been described in the vitreous 
body of humans and dogs with glaucoma. ${ }^{39}{ }^{40}$ Furthermore, it has been suggested that the raised glutamate levels that follow an ischaemic insult may contribute to neuronal toxicity and death of retinal ganglion cells. ${ }^{41}$ If ischaemia was already present in a proportion epilepsy patients before treatment, then raised neurotransmitter levels may be further altered by vigabatrin. Moreover, as the neuronal toxic effects of GABA are more enhanced in the retina compared to the rest of the CNS, patients with pre-existing ischaemia may be at a greater risk of suffering a retinal toxic effect from vigabatrin.

Whether the reduced ocular blood flow observed for the epilepsy patients when compared to the normal group is disease or AED related, cannot be determined from this study. Certainly the vigabatrin treated epilepsy group were more haemodynamically challenged compared to the conventionally treated epilepsy patients suggesting an underlying ischaemic mechanism specific to that group. Only a study incorporating a pre-AED and post-AED design will be able to elucidate whether the reduction in blood flow is disease or drug dependent.

Ocular perfusion is affected by numerous variables including cardiovascular factors and smoking. ${ }^{42}{ }^{43}$ All of the subjects recruited in this study were free from cardiovascular abnormalities, hypertension, and hypotension. None of the normal subjects smoked, however, one conventionally treated epilepsy patient and three vigabatrin treated epilepsy patients were smokers at the time of the study. In order to minimise variability between groups, all of the subjects refrained from smoking and nicotine substitutes, and underwent strict dietary control 24 hours before blood flow measurements. Pulsatile ocular blood flow is specifically influenced by IOP, pulse rate, and systemic blood pressure ${ }^{2644}$ and all three subject groups were matched for these variables.

Other factors reported to affect POBF include age, sex, axial length, refraction, scleral rigidity, posture, and vascular resistance. ${ }^{45-48}$ There was a difference in age between the groups; the mean age for the vigabatrin treated group was lower than both the normal control group and conventionally treated epilepsy group. It is known that POBF decreases with increasing $\mathrm{age}^{47}$ and, therefore, independent of all other factors, the vigabatrin group might have been expected to exhibit a higher mean POBF than the other two groups. In this study age differences were corrected for statistically by ANCOVA and the fact that the vigabatrin treated group had a lower POBF is supportive of an underlying mechanism that may be related to the drug or preselection of ischaemic individuals. While measurement of axial length was not incorporated into the study design, refractive error distributions were similar between groups (Table 1 ). There is currently no recognised measure for scleral rigidity, so this variable could not be accounted for. Subjects maintained a constant posture throughout measurement thus minimising posture related changes.

A previous study, that was based on a large cohort $(n=92)$ of vigabatrin treated patients reported a correlation between the extent of visual field damage and cumulative vigabatrin dosage. ${ }^{49}$ In contrast, a recent study that investigated the relation between the frequency of electrodiagnostic abnormalities and the duration of use or the total cumulative dosage of vigabatrin did not report any significant correlations. ${ }^{15}$ In this study, the investigation of potential correlations between POBF and PA, and vigabatrin maximum dose, duration, and cumulative dose did not yield significant correlations for any parameter. While mean ocular blood flow parameters differed between groups to ANCOVA, the small number of patients $(\mathrm{n}=10)$ contained within the vigabatrin treated epilepsy group meant that a high level of $r(>0.63)$ was required to demonstrate a significant correlation effect with drug dosage. It is likely, therefore, that with a larger cohort a significant effect may become apparent.

In conclusion, POBF and PA are reduced in patients with epilepsy undergoing AED therapy compared to normal healthy volunteers. The reduction is significantly more marked in those patients with a history of vigabatrin therapy. This may be as a direct consequence of the drug or disease, or it may suggest that a proportion of vigabatrin treated individuals are self selected for ischaemia. This reduction in ocular perfusion may have implications in the impairment of visual function associated with the drug. Further studies, particularly pretreatment and post-treatment investigations, are required to elucidate the mechanism of reduced ocular blood flow in patients treated with vigabatrin.

\section{ACKNOWLEDGEMENTS}

This work was funded in part by Aventis. We are grateful to Dr T Betts, FRCPsych, for his kind assistance with patient recruitment.

\section{Authors' affiliations}

S L Hosking, E J Roff Hilton, S J Embleton, Neurosciences Research Institute, Aston University, Aston Triangle, Birmingham B4 7ET, UK A K Gupta, City Hospital NHS Trust, Dudley Road, Birmingham B18 7QH, UK

\section{REFERENCES}

1 Theodore WH. Antiepileptic drugs and cerebral glucose metabolism. Epilepsia 1988.29(suppl 2):S48-55.

2 Matsuda H, Fukuchi t, Onuma T, et al. Interictal cerebral and cerebellar blood flow in temporal lobe epilepsy as measured by a non-invasive technique using Tc-99m HMPAO. Clin Nuc Med 1996;21:867-72.

3 Spanaki MV, Siegel H, Kopylev L, et al. The effect of vigabatrin (y-vinyl GABA) on cerebral blood flow and metabolism. Neurology 1999;53:1518-22.

4 Ludwig BL, Marsan CA. Clinical ictal patterns in epileptic patients with occipital electroencephalographic foci. Neurology 1975;25:463-71.

5 Steinhoff BJ, Freudenthaler N, Paulus W. The influence of established and new antiepileptic drugs on visual perception. II. A controlled study in patients with epilepsy under long-term antiepileptic medication. Epilepsy Res 1997;29:49-58.

6 Bayer A, Thiel HJ, Zrenner E, et al. Colour vision deficiencies and enhanced sensitivity to glare in epileptic patients treated with carbamazepine and phenytoin-ocular side-effects of anti-epileptic drugs. Nervenorzt 1995:66:89-96.

7 Nousiainen I, Kalviainen R, Mantyjarvi M. Color vision in epilepsy patients treated with vigabatrin or carbamazipine monotherapy. Ophthalmology 2000;107:884-8.

8 Eke T, Talbot JF, Lawden MC. Severe persistent visual field constriction associated with vigabatrin. BM 1997;314:180-1.

9 Krauss GL, Johnson MA, Miller NR. Vigabatrin-associated retinal cone system dysfunction. Neurology 1998;50:614-8.

10 Krauss G, Miller NR. Vigabatrin: an effective antiepilepsy drug-balancing the risk of visual dysfunction. Ann Pharmacother 1999;33:1367-8.

11 Manuchehri K, Goodman S, Siviter L, et al. A controlled study of vigabatrin and visual abnormalities. Br J Ophthalmol 2000;84:499-505.

12 Nousiainen I, Kalviainen R, Mantyjarvi M. Contrast and glare sensitivity in epilepsy patients treated with vigabatrin or carbamazepine monotherapy compared with healthy volunteers. Br J Ophthalmol 2000;84:622-5

13 Roff Hilton EJ, Cubbidge RP, Hosking SL, et al. Patients treated with vigabatrin exhibit central visual function loss. Epilepsia 2002; (in press).

14 Harding GFA, Robertson KA, Edson ASB, et al. Visual electrophysiological effect of a GABA transaminase blocker. Doc Ophthalmol 1999;97:179-88.

15 Coupland SG, Zackon DH, Leonard BC, et al. Vigabatrin effect on inner retinal function. Ophthalmology 2001;108:1493-6.

16 Fujiwara M, Muramatsu I, Shibata S. a-amino isobutyric acid recepto on vascular smooth muscle of dog cerebral arteries. Br J Pharmacol 1975;55:561-2.

17 Edvinsson L, Krause DN. Pharmacological characterization of GABA receptors mediating vasodilation of cerebral arteries in vitro. Brain Res 1979; 173:89-97

18 Kelly PT, McCulloch J. The effects of the GABAergic agonist muscimol upon the relationship between local cerebral blood flow and glucose utilization. Brain Res 1983;258:338-42.

19 Crooks J, Kolb H. Localisation of GABA, glycine and tyrosine-hydroxylase in the human retina. J Comp Neuro 1992;315:287-302.

20 Diamgoz MBA. Diversity of GABA receptors in the vertebrate outer retina. Trends Neurosci 1995;18:1 18-20.

21 Man-Kit Lam D. Neurotransmitters in the vertebrate retina. Invest Ophthalmol Vis Sci 1997;38:553-6.

22 Cubells JF, Blanchard JS, Smith DM, et al. In vivo action of enzyme-activated irreversible inhibitors of glutamic acid decarboxylase and gamma-aminobutyric acid transaminase in retina vs brain. $J$ Pharmacological Exp Ther 1986;238:508-14. 
23 Neal MJ, Shah MA. Development of tolerance to the effects of vigabatrin on GABA release from rat cerebral cortex, spinal cord and retina. $\mathrm{Br} J$ Pharmacol 1990; 100:324-8.

24 Commission on Classification and Terminology of the International League Against Epilepsy. Proposal for revised clinical and electronencephalographic classification of epileptic seizures. Epilepsia 1981;22:489-501.

25 Claridge KG, Smith SE. Diurnal variation in pulsatile ocular blood flow in normal and glaucomatous eyes. Surv Ophthalmol 1994;38:S198205.

26 Langham ME, Farell MA, O'Brien V. In: Lambrou GN, Greve EL, eds. Ocular blood flow in glaucoma. Non-invasive measurement of pulsatile ocular blood flow in the human eye. Amsterdam: Kugler and Ghedini Publications, 1989:93-9.

27 Krakau CET. Calculation of the pulsatile ocular blood flow. Invest Ophthalmol Vis Sci 1992;33:2754-6.

28 Silver DM, Farrell RA. Validity of pulsatile ocular blood flow measurements. Surv Ophthalmol.1994;38:572-80.

29 Langham ME, Farrell RA, O'Brien V, et al. Blood flow in the human eye Acta Ophthalmol 1989:67:9-13.

30 Silver DM, Farrell RA, Langham ME, et al. Estimation of pulsatile ocular blood flow from intraocular pressure. Acta Ophthalmol 1989;67:25-9.

31 Yang YC, Hulbert MFG, Batterbury M, et al. Pulsatile ocular blood flow measurements in healthy eyes:reproducibility and reference values. J Glaucoma 1997:6:175-9.

32 Spraul CW, Lang GE, Ronzani M, et al. Reproducibility of measurements with a new slit lamp-mounted ocular blood flow tonograph. Graefes Arch Clin Exp Ophthalmol 1998;236:274-9.

33 Spanaki MV, Siegel H, Kopylev L, et al. The effect of vigabatrin ( $\gamma$-viny GABA) on cerebral blood flow and metabolism. Neurology. 1999;53:1518-22.

34 Gasser P. Ocular vasospasm: a risk factor in the pathogenesis of low-tension glaucoma. Int Ophthalmol 1989;13:281-90.

35 O'Brien C, Butt Z. Blood flow velocity in the peripheral circulation of glaucoma patients. Ophthalmologica 1999;213:150-3.
36 Crofts K, Brennan R, O'Connor G, et al. Vigabatrin-induced optic neuropathy. J Neurol 1997;244:666-7.

37 Lawden MC, Eke T, Degg C, et al. Visual field defects associated with vigabatrin therapy. J Neurol Neurosurg Psychiatry 1999;67:716-22.

38 Osborne NN, Ugarte M, Chao M, et al. Neuroprotection in relation to ischemia and relevance to glaucoma. Surv Ophthalmol 1999:43:S102-28

39 Brooks DE, Garcia GA, Dreyer EB, et al. Vitreous body glutamate concentration in dogs with glaucoma. Am J Vet Res 1997;58:864-7.

40 Dreyer EB, Grosskreutz CL. Excitory mechanisms in retinal ganglion cell death in primary open angle glaucoma (POAG). Clin Neurosci 1997:4:270-3.

41 Sucher NJ, Lipton SA, Dreyer EB. Molecular basis of glutamate toxicity in retinal ganglion cells. Vis Res 1997;37:3483-93.

42 Steigerwalt R, Belcaro GV, Laurora G, et al. Ocular and orbital blood flow in patients with essential hypertension treated with trandolapril. Retina 1998; 18:539-45.

43 Lietz-Partzsch A, Griesser SM, Flammer J, et al. Decreased Heidelberg retina flowmeter (HRF) parameter "flow" at the papilla shortly after smoking a cigarette. Klin Studie 2000;218:332-4.

44 Vachon N, Kothe AC. Mechanisms mediating the postural effect of the pulsatile ocular blood flow. Optom Vis Sci 1992;69(Suppl):51-2.

45 Kothe AC, Vachon N, Woo S. Factors affecting pulsatile ocular blood flow:Axial length and ocular rigidity. Optom Vis Sci 1992;69 (Suppl):74.

46 Kothe AC. The effect of posture on intraocular pressure and pulsatile ocular blood flow in normal and glaucomatous eyes. Surv Ophthalmol 1994;38:S191-7

47 Ravalico G, Toffoli G, Pastori G, et al. Age-related ocular blood flow changes. Ophthalmology 1994;37:2645-50.

48 Silver DM, Geyer O. Pressure-volume relation for the living eye. Curr Eye Res 2000;20: 115-20.

49 Hardus P, Verduin WM, Engelsman M, et al. Visual field loss associated with vigabatrin: quantification and relation to dosage. Epilepsia 2001;42:262-7.

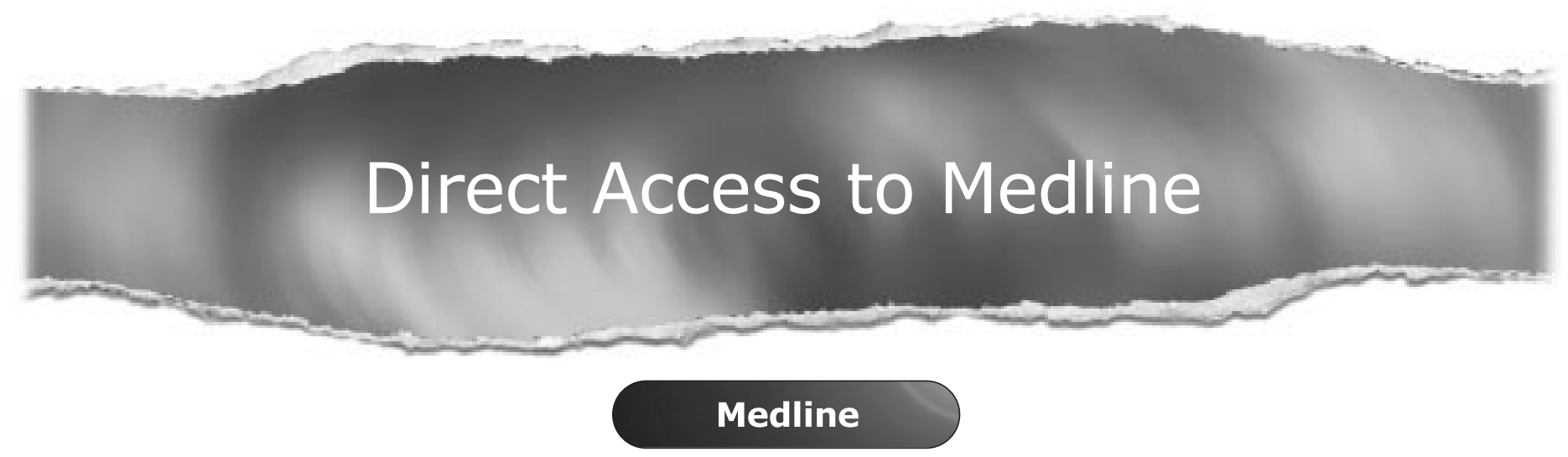

Link to Medline from the homepage and get straight into the National Library of Medicine's premier bibliographic database. Medline allows you to search across 9 million records of bibliographic citations and author abstracts from approximately 3,900 current biomedical journals.

\section{www.bjophthalmol.com}

